\title{
Chuva de sementes em uma floresta monodominante de Brosimum rubescens Taub. e em uma floresta mista adjacente no Vale do Araguaia, MT, Brasil ${ }^{1}$
}

\author{
Beatriz Schwantes Marimon ${ }^{2,3}$ e Jeanine Maria Felfili4,5
}

\author{
Recebido em 26/01/2005. Aceito em 27/09/2005
}

\begin{abstract}
RESUMO - (Chuva de sementes em uma floresta monodominante de Brosimum rubescens Taub. e em uma floresta mista adjacente no Vale do Araguaia, MT, Brasil). Manchas de Floresta monodominante de Brosimum rubescens ocorrem no ecótono entre o Cerrado e a Floresta Amazônica e encontram-se ameaçadas. Efetuou-se um estudo da chuva de sementes em duas porções de uma floresta monodominante de B. rubescens e uma floresta mista adjacente em Nova Xavantina, MT (1450'47'S e 5208'37' W). Partiu-se da premissa de que a floresta monodominante apresenta um padrão de chuva de sementes diferente da floresta mista, devido à maciça frutificação de B. rubescens. Em cada porção de floresta foram distribuídas aleatoriamente onze armadilhas $(0,5 \times 0,5 \mathrm{~m})$, totalizando 33 . Duas porções (22 armadilhas) estiveram na floresta monodominante e uma (11 armadilhas) na floresta mista e foram monitoradas entre agosto/2003 e julho/2004. As sementes foram separadas em duas categorias: dispersas pelo vento e dispersas por outro mecanismo, que não o vento, e as florestas foram comparadas em relação ao número de espécies, número de sementes e biomassa. Espécies com sementes pequenas apresentaram dispersão distribuída ao longo, enquanto sementes grandes, dispersão concentrada. A chuva de sementes foi influenciada pelo tipo de floresta e pelas estações do ano. Na floresta monodominante o pico da dispersão das sementes não dispersas pelo vento ocorreu no auge da estação seca e a maior parte da biomassa das sementes pertenceu a B. rubescens. A dispersão destas sementes no período seco pode conferir vantagens à espécie, diminuindo a competição inter-específica por agentes dispersores e/ou garantindo um recrutamento precoce. A produção de sementes grandes e em quantidade durante o período seco e a posterior formação de um extenso banco de plântulas podem ser algumas das estratégias (de ocupação de habitat) decisivas na monodominância desta espécie.
\end{abstract}

Palavras-chave: floresta monodominante, chuva de sementes, Brosimum rubescens, Amazônia, Cerrado

\begin{abstract}
Seed rain in a monodominant Brosimum rubescens Taub. forest and an adjacent mixed forest in the Araguaia River Valley, Mato Grosso State, Brazil). Patches of monodominant Brosimum rubescens forest occur at the transition zones between the Cerrado and the Amazonian Biomes. Seed rain was studied at two sites at the monodominant forest and another site at a nearby mixed

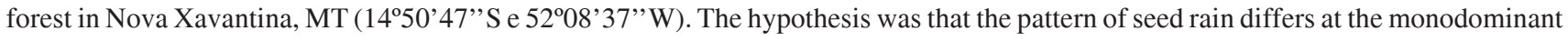
forest from the mixed forest due to the massive fruiting of Brosimum rubescens. Eleven seed-traps $(0,5 \times 0,5 \mathrm{~m})$ were set up at three portions of the forests, totalling 33 traps. Two portions ( 22 traps) were at the monodominant forest and one (11 traps) at a mixed forest. They were monitored from August 2003 to July 2004. The seed were classified as wind dispersed or dispersed by other mechanisms but not wind and the forests were compared regarding number and biomass of seeds and species richness. Number of seeds and biomass were compared at the three forest portions through the seasons. Wind dispersed, small seeded species were more evenly dispersed over the year that those big seed ones. Seed rain was affected by forest type and seasonality. At the monodominant forest the dispersion peak of non-wind dispersed seeds occurred at the dry season with most of the seed biomass belonging to B. rubescens. Abundant seed production, dispersion at the dry season and the development of a seedling bank, seems to be an advantage to the monodominant species. At the dry season the competition for dispersers is low, and the germination at the beginning of the rains allow the seedlings a full growth season.
\end{abstract}

Key words: monodominant forest, seed rain, Brosimum rubescens, Amazônia, Cerrado

\section{Introdução}

A regeneração natural das populações é condição fundamental para a manutenção de uma comunidade florestal (Templeton \& Levin 1979; Fenner 1985; Hofgaard 1993), processo que envolve, em maior ou menor grau fatores como produção, maturação e germinação de sementes, estabelecimento e

\footnotetext{
Parte da Tese de Doutorado da primeira Autora

2 Universidade de Brasília, Departamento de Ecologia, CEP 70919-970, Brasília, DF, Brasil

3 Universidade do Estado de Mato Grosso, Campus Universitário de Nova Xavantina, Departamento de Ciências Biológicas, C. Postal 08, CEP 78690-000, Nova Xavantina, MT, Brasil (biamarimon@ hotmail.com)

4 Universidade de Brasília, Departamento de Engenharia Florestal, C. Postal 04357, CEP 70900-900, Brasília, DF, Brasil

5 Autor para correspondência: felfili@unb.br
} 
sobrevivência de plântulas e a disponibilidade de um substrato adequado. A produção de sementes viáveis é fundamental para a regeneração (Fenner 1985) e a dispersão destas sementes é uma questão-chave na dinâmica da vegetação (Harper 1977). Swaine \& Whitmore (1988) observaram que as características das sementes podem moldar os padrões da história de vida das árvores tropicais, determinando onde e como a germinação e o estabelecimento podem ocorrer.

A chuva de sementes e o banco de sementes no solo são considerados indicadores importantes da capacidade de regeneração de uma floresta (Hopkins \& Graham 1983; Baider et al. 1999). O conhecimento da composição do banco de sementes é importante para compreender a dinâmica da vegetação, pois após o distúrbio de uma área natural a estrutura da vegetação será condicionada, num primeiro momento, pelas sementes das espécies presentes no solo (Campos \& Souza 2003). Por outro lado, a regeneração de uma floresta madura que tenha sofrido algum tipo de distúrbio (ex: abertura de uma clareira após a morte e tombamento de uma árvore) poderá depender, além de outros fatores importantes, da entrada de sementes na forma de chuva de sementes (Garwood 1989; Loiselle et al. 1996).

O conhecimento da variação da chuva de sementes ao longo do tempo contribui para a compreensão dos processos reprodutivos e da dinâmica de florestas naturais (Hofgaard 1993). Em florestas tropicais, os padrões sazonais de frutificação e a variação temporal no fluxo de propágulos (White 1994; Morellato 1995) podem ser fundamentais para determinar a ocupação de uma população em um habitat (Harper 1977). Neste contexto, o estudo da chuva de sementes pode fornecer informações importantes sobre a abundância, distribuição espacial e riqueza de espécies (Grombone-Guaratini \& Rodrigues 2002). Além disso, esta avaliação é importante, pois a dispersão espacial das sementes irá definir o modelo para o futuro padrão de regeneração e recrutamento da população (Loiselle et al. 1996).

Quando mais de 50\% do número de indivíduos ou da área basal de uma floresta pertencem a uma única espécie, esta é considerada uma "floresta monodominante" (Connell \& Lowman 1989; Hart et al. 1989). Em geral, estas florestas apresentam acentuado contraste com outras florestas tropicais com maior diversidade de espécies e representam um dos mais intrigantes enigmas da ecologia dos trópicos (Schluter \& Ricklefs 1993). Existem diversos exemplos de florestas tropicais monodominantes em solos bem drenados, em geral com florestas mistas adjacentes ocorrendo no mesmo tipo de solo (Hart et al. 1989; Martijena \& Bullock 1994; Torti et al. 2001; Henkel 2003; Newbery et al. 2004), incluindo um exemplo na Amazônia brasileira (Nascimento et al. 1997) e várias proposições já foram elaboradas para tentar explicar os mecanismos envolvidos na criação e manutenção destas florestas (Connell \& Lowman 1989; Torti et al. 2001).

A floresta monodominante de Brosimum rubescens Taub. (Moraceae) já foi estudada em relação à sua estrutura, composição florística, características edáficas e nutricionais (Felfili et al. 1998; Marimon \& Felfili 2000; Marimon et al. 2001a; b), mas ainda não foram desvendados todos os elementos relacionados com esta monodominância. Alguns estudos recentes têm sugerido que períodos episódicos de frutificação maciça poderiam contribuir para a manutenção das florestas monodominantes (Green \& Newbery 2002; Henkel 2003). A floresta de B. rubescens apresenta valor econômico, pois o cerne de sua principal espécie possui elevada durabilidade e é usado amplamente na região do Vale do Araguaia para a confecção de pilares, móveis, mourões, artesanato, etc, além de apresentar importância cultural para a comunidade indígena Xavante, que utiliza a madeira para a confecção de "bordunas" e os frutos para a alimentação e para atrair a caça (Marimon \& Felfili 2001). Intensas atividades de extração da madeira de $B$. rubescens, nos últimos anos, vêm contribuindo para o desaparecimento e descaracterização desta peculiar floresta.

Este estudo partiu da premissa de que a floresta monodominante apresenta um padrão de chuva de sementes diferenciado da floresta mista adjacente, com episódios de frutificação maciça de Brosimum rubescens.

O objetivo do presente estudo foi investigar a variação temporal e espacial na chuva de sementes em duas porções de uma floresta monodominante de Brosimum rubescens e uma porção de uma floresta mista adjacente. Foram investigados os efeitos dos tipos de floresta e das estações do ano no número de sementes, número de espécies e biomassa de sementes que compuseram a chuva de sementes no decorrer de 12 meses. Além disso, foi investigado se determinados mecanismos de dispersão estariam envolvidos com a queda de sementes nos diferentes tipos de floresta e estações do ano. A partir do histórico da chuva de sementes nas referidas florestas foram obtidas informações que podem contribuir para explicar a 
manutenção da monodominância de Brosimum rubescens.

\section{Material e métodos}

Área de estudo - O estudo foi desenvolvido entre os dias 5 de agosto/2003 e 20 de julho/2004 na floresta monodominante de Brosimum rubescens e na floresta mista adjacente, na Fazenda Vera Cruz (cujos antigos nomes mencionados em outros estudos foram: Fazenda Nova Viena (Felfili et al. 1998) e Fazenda Eldorado (Marimon et al. 2001a; Marimon \& Felfili 2001), retiro Pau Brasil, município de Nova Xavantina, MT, situadas a $14^{\circ} 50^{\prime} 47^{\prime}$ 'S e $52^{\circ} 08^{\prime} 37^{\prime}$ 'W. O clima da região é do tipo Aw, segundo a classificação de Köppen, com uma estação seca e uma chuvosa bem definidas e precipitação média anual de $1.500 \mathrm{~mm}$ (Marimon et al. 2001a). No entorno da área estudada ocorre um mosaico de formações florestais, onde a floresta monodominante constitui uma extensa mancha, seguida da floresta mista, floresta de transição, floresta de galeria pantanosa, entre outros (Felfili et al. 1998; Marimon 1998; B.S. Marimon, dados não publicados).

Foram escolhidas duas porções de floresta monodominante, uma com elevada concentração de árvores adultas (DAP>25 cm) de B. rubescens, caracterizada por apresentar mais de sete indivíduos por $100 \mathrm{~m}^{2}$ (Monodominante Rica - Mono-R) e outra porção caracterizada por apresentar menos de três indivíduos adultos por $100 \mathrm{~m}^{2}$ (Monodominante Pobre - Mono-P), ambas se enquadrando na definição de floresta monodominante proposta por Connell \& Lowman (1989) e Hart et al. (1989). A floresta mista adjacente (distante cerca de $800 \mathrm{~m}$ da floresta monodominante) caracterizou-se por apresentar maior riqueza de espécies (43 espécies na monodominante e 84 na mista) e apenas dois indivíduos adultos de B. rubescens ocorreram nas proximidades da área abrangida pelas armadilhas de coleta de sementes.

Coleta de dados - Nas florestas monodominante e mista foram demarcadas 60 parcelas contíguas (em cada) de $10 \times 10$ m e em cada porção de floresta (Mono$\mathrm{R}$, Mono-P e Mista) foram distribuídas aleatoriamente, com uma distância mínima de $5 \mathrm{~m}$, onze armadilhas $(0,5 \mathrm{~m} \times 0,5 \mathrm{~m} \times 0,5 \mathrm{~m})$, totalizando 33 , construídas a partir de uma estrutura de madeira com uma rede de tela plástica (1 mm) formando uma bolsa piramidal invertida. Entre 5 de agosto/2003 e 20 de julho/2004, a cada 15 dias, o material depositado nas armadilhas foi coletado, acondicionado em sacos de papel e secado em estufa $\left(70{ }^{\circ} \mathrm{C}\right)$ até peso constante. Posteriormente, com o auxílio de uma lupa de mesa, todas as sementes aparentemente saudáveis (sem sinais de apodrecimento ou predação) e viáveis foram contadas, separadas, pesadas em balança analítica e identificadas. As sementes foram identificadas ao nível taxonômico mais baixo possível a partir da coleta de frutos e comparações com o material depositado no Herbário NX, Campus Universitário de Nova Xavantina e no Herbário UB, Universidade de Brasília.

Os dados de precipitação $(\mathrm{mm})$ foram coletados na Estação Meteorológica de Nova Xavantina (Ministério da Agricultura, $9^{\circ}$ Distrito de Meteorologia) localizada no Campus Universitário de Nova Xavantina distante cerca de $20 \mathrm{~km}$ (em linha reta) da área de estudo. Os meses de chuva foram aqueles que apresentaram, no mínimo, $150 \mathrm{~mm}$ de precipitação. A precipitação mensal máxima registrada na época da seca (no período de estudo) foi de $63,8 \mathrm{~mm}$, no mês de outubro. No período estudado, as temperaturas média anual, mínima e máxima foram $24,7{ }^{\circ} \mathrm{C}, 19,1{ }^{\circ} \mathrm{C}$ e $32,7^{\circ} \mathrm{C}$, respectivamente e a precipitação foi de $1.642 \mathrm{~mm}$ (Fig.1, precipitação).

Análise dos dados - As sementes foram separadas em duas categorias: dispersas pelo vento e dispersas por outro mecanismo (não-vento), baseando-se na presença de estruturas de vôo na semente ou no diásporo. A abundância temporal das sementes foi expressa a partir do número de espécies, número de sementes e biomassa das sementes, calculadas por período de coleta por $\mathrm{m}^{2}$. Cada período de coleta (a cada 15 dias) foi classificado como pertencente à época da chuva (novembro a abril) ou à época da seca (maio a outubro).

Foram registradas as informações temporais do número e da biomassa das sementes de Brosimum rubescens nas porções de floresta monodominante (Mono-R e Mono-P) e foi também registrada a fenologia da queda das sementes de Amaioua intermedia, Chaetocarpus echinocarpus, Protium pilosissimum e Tetragastris altissima, espécies de fácil identificação e de destaque na estrutura fitossociológica da floresta monodominante e/ou da floresta mista adjacente (Marimon et al. 2001a). A. intermedia apresentou densidade absoluta de 128 indivíduos/hectare na floresta monodominante e 127 na mista, C. echinocarpus apresentou 3 e 45, P. pilosissimum, 163 e 45 e T. altissima, 70 e 65, respectivamente na floresta monodominante e na mista (B.S. Marimon, dados não publicados). 
Foi utilizado o teste não-paramétrico de KruskalWallis para comparar o número de sementes (dispersas pelo vento e por outro mecanismo, que não o vento) e o número de espécies (dispersas pelo vento e por outro mecanismo), no período das chuvas e da seca, nas três porções de floresta. Quando foram encontradas diferenças significativas utilizou-se o teste de Dunn (Zar 1999) para determinar os grupos. O número e a biomassa das sementes de Brosimum rubescens, que só ocorreu nas duas porções de floresta monodominante, foram comparados entre as porções de floresta (Mono-R e Mono-P) e para cada porção de
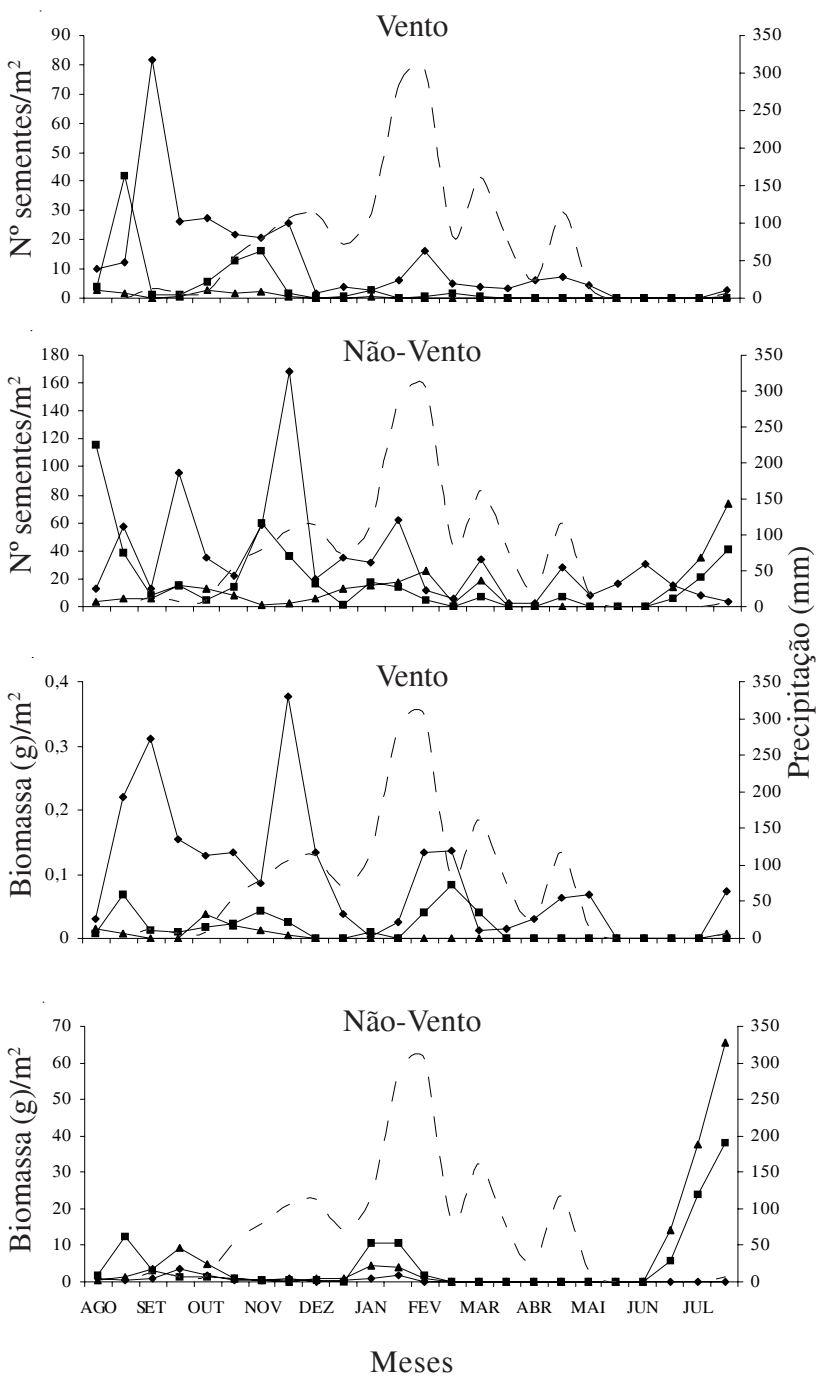

Figura 1. Queda de sementes (número de sementes $/ \mathrm{m}^{2}$ e biomassa $\mathrm{g} / \mathrm{m}^{2}$ ) dispersas pelo vento e por outros mecanismos (não-vento) $\mathrm{e}$ precipitação $(\mathrm{mm})$, de agosto/2003 a julho/2004, na floresta monodominante de B. rubescens (Mono- $\mathrm{R}=$ Monodominante Rica e Mono-P = Monodominante Pobre) e na floresta mista adjacente (Mista), Nova Xavantina, MT. - - = Mista; - - $-=$ Mono-P; $-\mathbf{\Delta}-=$ Mono-R; $\cdots \cdots . . .=$ Precipitação. floresta, entre as estações do ano (seca e chuva), através do teste de Mann-Whitney.

Os dados de todos os parâmetros analisados foram avaliados com relação à normalidade (teste de Kolmogorov-Smirnov) e homocedasticidade (teste de Bartlett). Somente os dados de biomassa, que foram log-transformados, atenderam aos pressupostos exigidos para efetuar uma análise de variância (Zar 1999). Assim, para os dados de biomassa das sementes foi utilizada uma análise de variância fatorial para examinar a importância das porções de floresta e das estações do ano na determinação da biomassa nos diferentes períodos de coleta. As variáveis dependentes foram a biomassa das sementes dispersas pelo vento e a biomassa das sementes dispersas por outro mecanismo (não-vento) e as variáveis independentes foram os tipos de floresta (Mono-R, Mono-P e Mista) e as estações do ano (Seca e Chuva). Todas as análises estatísticas foram feitas a partir do Programa SAS v. 8.1 (SAS 1999-2000).

\section{Resultados}

Uma média de 288 sementes $/ \mathrm{m}^{2}$ caíram nas armadilhas da floresta Mono-R, $518 \mathrm{sem} . / \mathrm{m}^{2}$ na floresta Mono-P e 1.067 sementes $/ \mathrm{m}^{2}$ na Mista, no período de estudo de 12 meses.

Um total de 5.155 sementes foi encontrado nas armadilhas nas três porções de floresta durante os 12 meses de coleta. Deste total, 1.082 sementes caracterizaram-se como dispersas pelo vento, concentrando-se 3\% na floresta Mono-R, 23\% na Mono-P e 74\% na Mista e 4.073 sementes como dispersas por outro mecanismo que não o vento, sendo $18 \%, 29 \%$ e $53 \%$ na floresta Mono-R, Mono-P e Mista, respectivamente.

As sementes coletadas nas três porções de floresta foram separadas em 59 morfo-espécies, sendo 27 dispersas pelo vento e 32 por não-vento. Nas armadilhas da floresta Mono- $\mathrm{R}$ foram coletadas 21 morfo-espécies, sendo Vento $=9$ e Não-Vento $=12$, na floresta Mono-P foram, $\mathrm{V}=11$ e NV $=12$ e na Mista, $\mathrm{V}=21$ e $\mathrm{NV}=19$. Foram encontradas diferenças significativas (Kruskal-Wallis, $\chi^{2}=15,41, \mathrm{P}=0,0005$ ) no número de espécies dispersas pelo vento entre as três porções de floresta, sendo que a floresta Mista apresentou o maior número de espécies. Entretanto, não houve diferença estatisticamente significativa, entre as três porções de floresta $(\mathrm{P}>0,05)$, no número de espécies dispersas por outro mecanismo que não o vento. 
Os resultados da análise de variância fatorial revelaram a importância do tipo de floresta e das estações do ano como influência no padrão da chuva de sementes (Tab. 1). Entretanto, a importância relativa destes dois fatores diferiu entre o tipo de dispersão de sementes (vento e não-vento). A biomassa das sementes dispersas pelo vento foi afetada pelo tipo de floresta, mas não foi afetada pelas estações do ano. Por outro lado, a biomassa das sementes de não-vento foi afetada pelos tipos de floresta, pelas estações do ano e pela interação de ambas. Provavelmente, neste caso, o fator determinante foi a biomassa das sementes de Brosimum rubescens, a espécie dominante das florestas Mono-R e Mono-P.

$\mathrm{O}$ número de sementes dispersas pelo vento no período estudado foi marcadamente sazonal na floresta Mono-P e na Mista, com um acentuado pico no final da estação seca e outro menor no início das chuvas (Fig. 1, vento). O padrão de variação sazonal das sementes dispersas pelo vento diferiu entre o número e a biomassa. O número de sementes dispersas pelo vento apresentou maior paridade entre as estações seca e chuvosa (elevado número de sementes na seca e reduzido na chuvosa) e a biomassa apresentou uma variação mais homogênea no decorrer do ano. Estas diferenças foram caracterizadas, principalmente, pelo tamanho e biomassa das sementes que variaram sua dispersão no decorrer do período.

O número de sementes dispersas pelo vento apresentou diferenças significativas entre a floresta monodominante e a floresta mista (Teste de KruskalWallis, $\left.\chi^{2}=22,56, \mathrm{P}<0,0001\right)$, principalmente no

Tabela 1. Resultados da análise de variância fatorial (Model III) testando os efeitos do tipo de floresta (Mista, Monodominante Pobre e Monodominante Rica) e das estações do ano (seca e chuva) na biomassa das sementes dispersas pelo vento e dispersas por outros mecanismos (não-vento), coletadas em 24 períodos no decorrer de um ano. Os dados foram log-transformados para a análise.

\begin{tabular}{lrrr}
\hline & g.l. & F & \multicolumn{1}{c}{$\mathrm{P}$} \\
\hline Vento & & & \\
$\quad$ Floresta & 2 & 12,77 & $<0,0001$ \\
$\quad$ Estações & 1 & 4,07 & 0,0512 \\
$\quad$ Floresta*Estações & 2 & 1,30 & 0,2861 \\
Erro & 36 & & \\
Não-Vento & & & \\
Floresta & 2 & 5,49 & 0,0067 \\
Estações & 1 & 15,04 & 0,0003 \\
Floresta*Estações & 2 & 3,22 & 0,0475 \\
Erro & 55 & & \\
\hline
\end{tabular}

período das chuvas. Na floresta Mono-P e na Mista, a biomassa das sementes dispersas pelo vento apresentou um pico no final da seca e outro no início das chuvas (Fig. 1, vento). As sementes dispersas pelo vento no final da seca caracterizaram-se por apresentar menor biomassa e número de indivíduos (ex: Uncaria guianensis e Arrabidaea spp.) e as sementes dispersas pelo vento no início das chuvas caracterizaram-se por uma maior biomassa e um menor número (ex: Hippocratea volubilis e Heteropterys eglandulosa) (Fig. 1, vento).

$\mathrm{Na}$ floresta Mista o número de sementes dispersas por outros mecanismos que não o vento apresentou diversos picos no decorrer do período estudado, sendo o maior no início das chuvas e os demais geralmente coincidindo com picos na precipitação (Fig. 1, não-vento). Este padrão de picos frequientes no decorrer do ano pode estar associado à maior diversidade de espécies registrada na floresta Mista. Na floresta Mono-P foi observado um pico no auge da seca e outro no início das chuvas e na Mono-R só houve um pico acentuado no auge da seca, justamente quando na floresta Mista os valores foram mínimos (Fig. 1, não-vento). O número de sementes dispersas por não-vento apresentou diferenças significativas entre as três porções de floresta (Kruskal-Wallis, $\chi^{2}=9,96$, $\mathrm{P}=0,0069$ ), sendo a Mono-P e a floresta Mista similares entre si, especialmente no período chuvoso.

De forma geral, o padrão de variação sazonal observado no número de sementes dispersas por nãovento se repetiu na biomassa das sementes, também dispersas por não-vento. O número de sementes dispersas por não-vento registrado na floresta Mista apresentou uma variação homogênea no período estudado, com alguns picos de dispersão. Entretanto, os valores de biomassa foram quase desprezíveis quando comparados com a floresta monodominante (Mono-R e Mono-P), caracterizando-se assim por apresentar sementes menores. Observou-se também que na biomassa das sementes dispersas por não-vento os maiores picos foram caracterizados pelas sementes das florestas Mono-R e Mono-P (Fig. 1, não-vento). Neste caso, foram as sementes de Brosimum rubescens que predominaram e definiram os valores da biomassa em ambas as porções de floresta monodominante, visto que esta espécie não foi registrada nas armadilhas da floresta mista (Fig. 2). Considerando-se a biomassa total de sementes dispersas por não-vento, observou-se que a proporção entre as três florestas foi de 1:8:11 gramas entre a Mista, Mono-P e Mono-R, respectivamente. 
Comparando-se apenas o número e a biomassa das sementes de Brosimum rubescens, entre as florestas Mono-R e Mono-P, verificou-se que os valores sempre foram significativamente similares (Teste de Mann-Whitney) entre as duas porções de floresta. Entretanto, ao analisarem-se as porções separadamente foi verificada uma diferença significativa entre as estações do ano, sendo que o número e a biomassa das sementes de $B$. rubescens sempre foram superiores no período da seca, tanto na Mono-R quanto na Mono-P (Teste de Mann-Whitney, $\mathrm{U}>110, \mathrm{P}<0,05$ ).
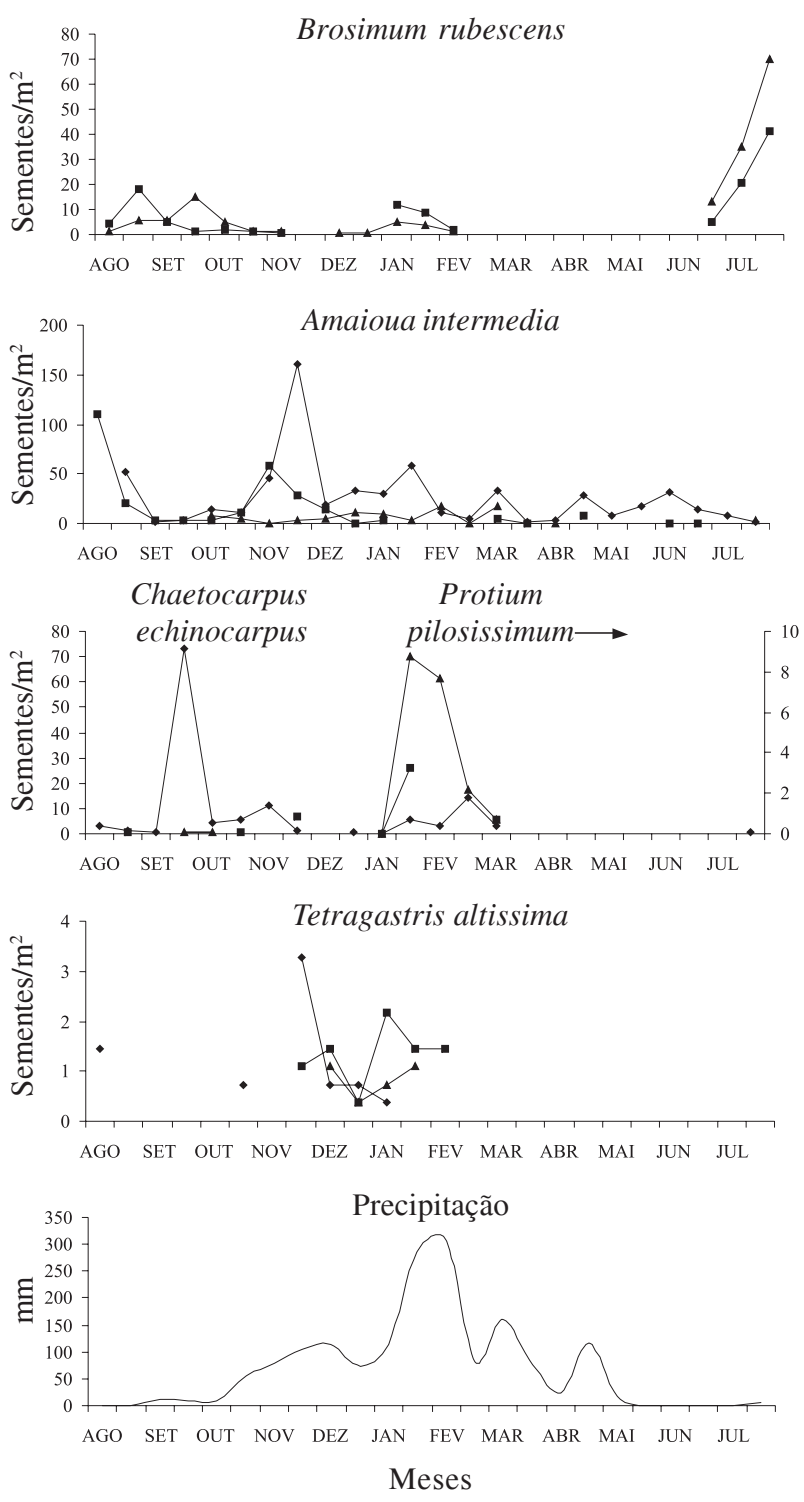

Figura 2. Fenologia da queda de sementes das principais espécies da floresta monodominante de $B$. rubescens $($ Mono- $\mathrm{P}=$ Monodominante Pobre e Mono-R = Monodominante Rica) e da floresta mista adjacente (Mista) nos diferentes períodos de coleta em Nova Xavantina, MT. $---=$ Mista; $---=$ Mono-P; $-\mathbf{-}-=$ Mono-R.
Observando-se a fenologia da queda de sementes de quatro espécies importantes nas três porções de floresta estudadas, foram identificados alguns padrões distintos (Fig. 2). Amaioua intermedia apresentou sementes pequenas (biomassa seca, média $=3,5 \mathrm{mg}$; 0,2 a $0,4 \mathrm{~cm}$ de comprimento) que estiveram distribuídas em um padrão relativamente constante no decorrer do ano. Por outro lado, Chaetocarpus echinocarpus $(5,1 \mathrm{mg} ; 0,5$ a $0,8 \mathrm{~cm})$, Protium pilosissimum (38mg; 0,8 a $1 \mathrm{~cm})$ e Tetragastris altissima ( $88 \mathrm{mg} ; 1$ a $1,5 \mathrm{~cm})$, apresentaram sementes maiores (1,5 a 25 vezes) do que a primeira espécie, maior concentração sazonal e menores períodos de dispersão das sementes. Brosimum rubescens $(900 \mathrm{mg}$; 1 a $2,5 \mathrm{~cm}$ ), cujas sementes estão entre as maiores dentre as espécies registradas nas florestas monodominante e mista, apresentou uma combinação entre o padrão identificado para a espécie de sementes pequenas (A. intermedia) e aquele observado para as demais espécies, com sementes maiores, com um pico acentuado em um período específico (auge da seca) e picos menores em outros períodos ao longo do ano (Fig. 2).

\section{Discussão}

A densidade de sementes coletadas no período de estudo (Mono- $\mathrm{R}=0,8$ sementes $/ \mathrm{m}^{2} / \mathrm{dia}$, Mono- $\mathrm{P}=1,44$ e Mista $=2,96)$ esteve dentro da amplitude encontrada em outras florestas tropicais, cujos valores podem ser bastante variáveis, refletindo diferenças metodológicas ou diferenças específicas de cada comunidade estudada (Grombone-Guaratini \& Rodrigues 2002). Dentre outros, Walker \& Neris (1993) registraram valores que variaram de 0,3 , nas áreas abertas, até $8,8 \mathrm{sem} . / \mathrm{m}^{2} / \mathrm{dia}$, em áreas de floresta fechada em Porto Rico, dois anos após a passagem do furacão Hugo. Young et al. (1987) encontraram $5,8 \mathrm{sem} . / \mathrm{m}^{2} /$ dia em uma floresta secundária da Costa Rica, Denslow \& Gomez-Diaz (1990) registraram $1,6 \mathrm{sem} . / \mathrm{m}^{2} / \mathrm{dia}$ em uma floresta tropical, também na Costa Rica e Clark \& Poulsen (2001) encontraram cerca de $0,6 \mathrm{sem} . / \mathrm{m}^{2} /$ dia em uma floresta tropical semi-decídua em Camarões.

A marcante sazonalidade, observada no presente estudo para as sementes dispersas pelo vento, esteve de acordo com outros estudos realizados em florestas tropicais onde as sementes dispersas pelo vento predominaram durante a estação seca e no início da estação chuvosa, tal como observado por Jackson (1981) no Espírito Santo, Grombone-Guaratini \& Rodrigues (2002) em São Paulo, Foster (1985) na Ilha 
de Barro Colorado no Panamá e por Carrière et al. (2002) em Camarões. Na seca, a maior deciduidade das espécies e a reduzida precipitação podem garantir uma dispersão mais eficiente das sementes anemocóricas (Morellato 1995).

A dispersão de sementes por outros mecanismos que não o vento não apresentou uma sazonalidade marcante, sendo que a floresta Mista foi a que mais se aproximou dos resultados registrados em outras florestas tropicais, apresentando um pico no início da estação chuvosa e outros picos menores no decorrer desta estação. Jackson (1981), Foster (1985), Holl (1999) e Grombone-Guaratini \& Rodrigues (2002) verificaram que a disseminação das sementes dispersas por outros mecanismos que não o vento (em geral, zoocoria) ocorria no início ou no decorrer da estação chuvosa.

Os resultados observados na floresta monodominante (Mono-R e Mono-P) foram distintos daqueles registrados na floresta Mista, pois o pico de dispersão das sementes dispersas por não-vento (número e biomassa) ocorreu no auge da estação seca. Neste caso, a maior parte do número e da biomassa das sementes pertenceu à espécie monodominante, Brosimum rubescens (na floresta Mono-R, das 148,7 g de sementes $/ \mathrm{m}^{2} /$ ano coletadas, $147 \mathrm{~g}$ pertenceram a B. rubescens e na porção Mono-P, de um total de $113,3 \mathrm{~g}, 109,6 \mathrm{~g}$ representavam sementes de B. rubescens), sugerindo a possibilidade da manutenção da dominância.

A dispersão destas sementes no período seco pode conferir vantagens à espécie monodominante, diminuindo a competição inter-específica por agentes dispersores ou garantindo um recrutamento precoce. Jackson (1981), estudando uma floresta sub-tropical no Espírito Santo, verificou que algumas espécies dispersas por não-vento apresentavam frutificação no início da estação seca.

No presente estudo, Amaioua intermedia, com sementes pequenas, apresentou uma dispersão distribuída ao longo de todo o ano e as demais espécies, com sementes maiores, apresentaram uma dispersão mais concentrada. Smythe (1970) observou que a dispersão de sementes maiores (maior biomassa) estaria distribuída de forma mais concentrada do que a dispersão de sementes menores, sendo que este padrão indicaria uma fundamental diferença na pressão seletiva sobre espécies de sementes grandes e espécies de sementes pequenas. As espécies de sementes grandes seriam beneficiadas por uma frutificação mais sincronizada, proporcionando um maior acúmulo de sementes e as sementes pequenas, que em geral são dispersas por endozoocoria, seriam beneficiadas por uma dispersão mais distribuída ao longo do ano. Jackson (1981) encontrou resultados similares, registrou períodos de frutificação mais longos para as espécies de sementes pequenas e sugeriu que para as espécies de sementes grandes, cuja frutificação foi mais concentrada, a saciedade do predador de sementes seria um importante mecanismo na regulação do consumo de frutos.

É possível supor que a estrutura atual da floresta monodominante de Brosimum rubescens seja mantida por seu padrão de regeneração em nível temporal e espacial. Allmen et al. (2004) observaram que sob condições de elevada densidade de sementes, como as registradas para Euterpe edulis, escapar do predador seria mais uma questão de quando as sementes são dispersas do que onde são depositadas. Por outro lado, Schupp (1992) e Chapman \& Chapman (1996) observaram que, no contexto populacional, o número de sementes sobreviventes pode aumentar com a densidade de árvores adultas em função da saciedade do predador, tal como foi observado por Hart (1995), que registrou menor predação de sementes de espécies dominantes em áreas de floresta monodominante do que em áreas de florestas mistas. Experimentos realizados por Boucher (1981) demonstraram que a saciedade de predadores de sementes poderia explicar a manutenção e não o estabelecimento da dominância de uma espécie.

Considerando-se que a produção de sementes de B. rubescens é maciça e que as sementes estão entre as maiores (maior biomassa) da comunidade, a capacidade das sementes sobreviverem nas proximidades dos indivíduos adultos pode estar associada a diversos aspectos da história de vida desta espécie. Levey (1987) observou que animais frugívoros em geral evitam carregar sementes grandes muito longe das árvores que estão frutificando e, da mesma forma, Foster \& Janson (1985) verificaram que sementes grandes aumentam o vigor das plântulas que crescem próximas à planta-mãe. Martinez-Ramos \& SotoCastro (1993) também observaram que um maior investimento parental (produção de sementes grandes) resultaria em uma maior probabilidade de sobrevivência da plântula sendo que, espécies que produzem sementes grandes teriam maiores chances de serem representadas abundantemente na fase de plântula e consequientemente em estágios posteriores de seu ciclo vital. Hart et al. (1989) observaram que espécies monodominantes tendem a apresentar sementes 
grandes, plântulas resistentes e reduzida capacidade de dispersão.

Alguns estudos discutiram o papel da limitação de recrutamento como sendo um mecanismo importante para manter a elevada diversidade de espécies em comunidades tropicais (Tilman 1994; 1999; Hubbell et al. 1999; Webb \& Peart 2001; Wang \& Smith 2002). Neste caso, entende-se como limitação de recrutamento a dispersão limitada de sementes em um espaço e a fecundidade limitada da planta-mãe, quando não são produzidas sementes em número suficiente (Webb \& Peart 2001). De acordo com Hubbell et al. (1999), a limitação de recrutamento explicaria a coexistência de um grande número de espécies que apresentam necessidades similares de recursos, tal como ocorre nas florestas tropicais úmidas. Assim, devido a uma limitada dispersão de sementes e um limitado recrutamento, determinados competidores superiores poderiam não alcançar certos locais e permitiriam que competidores inferiores vencessem "por falta" (Tilman 1999).

Brosimum rubescens apresenta sementes grandes e grande produção de sementes (grande investimento parental), abundante banco de plântulas no sub-bosque (Marimon et al. 2001a) e a concentração da produção de sementes em um período desfavorável (seca) para a maioria das espécies. Neste caso, não apresentaria limitação de recrutamento e sim um eficiente e agressivo mecanismo de competição, o que garantiria a sua dominância na comunidade. Gouveia \& Felfili (1998) observaram que as espécies mais abundantes em uma floresta de galeria em Brasília, DF amadureceram os frutos na estação seca e esta estratégia garantiria a dominância destas espécies, pois as sementes apresentariam melhores chances de germinação e estabelecimento com o início das chuvas.

Se o padrão atual de regeneração de B. rubescens sofrer alguma mudança no decorrer dos anos a estrutura da população também deverá sofrer mudanças, assim como a comunidade, pois a dominância de poucas espécies na chuva de sementes pode afetar, em longo prazo, a dinâmica de uma floresta (Cubiña \& Aide 2001). Entretanto, uma ampla escala temporal pode ser necessária para a alteração de estruturas de comunidades e populações, pois como foi observado por Runkle (1989), as consequiências reprodutivas de anos com boa regeneração podem garantir a sobrevivência da população durante os maus anos. Conseqüentemente, o sucesso de uma boa regeneração periódica poderia ser suficiente para manter a estrutura da floresta estudada durante muitas gerações.

O presente estudo forneceu informações sobre a perspectiva de regeneração das florestas avaliadas, indicando que a chuva de sementes foi afetada pelo tipo de floresta, pelas estações do ano e pela combinação de ambas. A chuva de sementes da floresta monodominante diferiu do padrão observado na floresta mista adjacente. Nas porções de floresta monodominante a predominância de sementes de Brosimum rubescens (frutificação maciça) pode representar um importante papel na estrutura e dinâmica desta comunidade e na manutenção da dominância desta espécie. Além disso, tal como foi observado por Hamrick et al. (1993) e Lepsch-Cunha et al. (1999), os mecanismos de dispersão das sementes e a densidade dos indivíduos adultos da espécie monodominante podem apresentar um impacto direto na estrutura e variabilidade genética da população e representar importantes implicações para a conservação da espécie in situ e ex situ. Estudos mais detalhados são imprescindíveis para confirmar este padrão.

As características das sementes de B. rubescens e a elevada capacidade de autoregeneração associada à ausência de limitação de recrutamento podem ser fatores decisivos na manutenção da monodominância desta espécie. As características que diferenciam as três porções de floresta estudadas estão relacionadas aos eventos de dispersão predominantes em cada tipo de floresta. Tais eventos foram delineados a partir de uma variação espacial na disponibilidade de sementes (ex: floresta monodominante apresentou maior biomassa de sementes dispersas por não-vento) e a partir de uma variação nos padrões fenológicos (ex: floresta Mista apresentou um forte padrão sazonal das espécies dispersas pelo vento). Assim, a interação entre ambos afetaria os padrões envolvidos nas atividades dos animais dispersores e na disseminação das sementes e determinaria, em longo prazo, a estrutura e a dinâmica destas comunidades florestais.

\section{Agradecimentos}

As autoras agradecem à CAPES e ao CNPq, pelas bolsas concedidas; ao professor Ben Hur Marimon Júnior, ao biólogo Herson Souza Lima e aos acadêmicos do curso de Ciências Biológicas da UNEMAT-Nova Xavantina, pelo auxílio na coleta de dados. 


\section{Referências bibliográficas}

Allmen, C.; Morellato, P.C. \& Pizo, M.A. 2004. Seed predation under high seed density condition: the palm Euterpe edulis in the Brazilian Atlantic Forest. Journal of Tropical Ecology 20: 471-474.

Baider, C.; Tabarelli, M. \& Mantovani, W. 1999. O banco de sementes de um trecho de floresta atlântica montana (São Paulo, Brasil). Revista Brasileira de Botânica 59(2): 319-328.

Boucher, D.H. 1981. Seed predation by mammals and forest dominance by Quercus oleoides, a tropical lowland oak. Oecologia 49: 409-414.

Campos, J.B. \& Souza, M.C. 2003. Potencial for natural forest regeneration from seed bank in an upper Paraná River floodplain, Brazil. Brazilian Archives of Biology and Technology 46(4): 625-639.

Carrière, S.M.; André, M.; Letourmy; P., Olivier, I. \& McKey, D.B. 2002. Seed rain beneath remnant trees in a slashand-burn agricultural system in southern Cameroon. Journal of Tropical Ecology 18: 353-374.

Chapman, C.A. \& Chapman, L.J. 1996. Frugivory and the fate of dispersed and non-dispersed seeds of six African tree species. Journal of Tropical Ecology 12: 491-504.

Clark, C.J. \& Poulsen, J.R. 2001. The role of arboreal seed dispersal groups on the seed rain of a lowland tropical forest. Biotropica 33(4): 606-620.

Connell, J.H. \& Lowman, M.D. 1989. Low-diversity tropical rain forests: some possible mechanisms for their existence. The American Naturalist 134: 88-119.

Cubiña, A. \& Aide, M. 2001. The effect of distance from forest edge on seed rain and soil seed bank in a tropical pasture. Biotropica 33(2): 260-267.

Denslow, J.S. \& Gomez-Diaz, A.E. 1990. Seed rain to tree-fall gaps in a neotropical rain forest. Canadian Journal of Forest Research 20: 642-648.

Felfili, J.M.; Silva Júnior, M.C. \& Nogueira, P.E. 1998. Levantamento da vegetação arbórea na região de Nova Xavantina, MT. Boletim do Herbário Ezechias Paulo Heringer 3: 63-81.

Fenner, M. 1985. Seed ecology. New York, Chapman \& Hall.

Foster, R.B. 1985. The seasonal rhythm of fruitfall on Barro Colorado Island. Pp. 151-172. In: E.G. Leigh; A.S. Rand \& D.M. Windsor (eds.). The Ecology of a Tropical Forest. Washington, DC., Smithsonian Institution Press.

Foster, S.A. \& Janson, C.H. 1985. The relationship between seed size and establishment conditions in tropical woody plants. Ecology 66: 773-780.

Garwood, N.C. 1989. Tropical soil seed banks: a review. Pp. 149-208. In: M.A. Leck; V.T. Parker \& R.L. Simpson (eds.). Ecology of soil seed banks. San Diego, Academic Press.

Gouveia, G.P. \& Felfili, J.M. 1998. Fenologia de comunidades de Mata de Galeria e de Cerrado no Distrito Federal. Revista Árvore 22(4): 443-450.

Green, J.J. \& Newbery, D.M. 2002. Reproductive investment and seedling survival of the mast-fruiting rain forest tree, Microberlinia bisulcata A. chev. Plant Ecology 162: $169-183$.
Grombone-Guaratini, M.T. \& Rodrigues, R.R. 2002. Seed bank and seed rain in a seasonal semi-deciduous forest in south-eastern Brazil. Journal of Tropical Ecology 18: 759-774.

Hamrick, J.L.; Murawski, D.A. \& Nason, J.D. 1993. The influence of seed dispersal mechanisms on the genetic structure of tropical tree populations. Vegetatio 107/108: 281-297.

Harper, J.L. 1977. Population biology of plants. London, Academic Press.

Hart, T.B. 1995. Seed, seedling and sub-canopy survival in monodominant and mixed forests of the Ituri Forest, Africa. Journal of Tropical Ecology 11: 443-459.

Hart, T.B.; Hart, J.A. \& Murphy, P.G. 1989. Monodominant and species-rich forests of the humid tropics: causes for their co-occurrence. The American Naturalist 133(5): 613-633.

Henkel, T.W. 2003. Monodominance in the ectomycorrhizal Dicymbe corymbosa (Caesalpiniaceae) from Guyana. Journal of Tropical Ecology 19: 417-437.

Hofgaard, A. 1993. Seed rain quantity and quality, 19841992, in a high altitude old-growth spruce forest, northern Sweden. New Phytologist 125: 635-640.

Holl, K.D. 1999. Factors limiting tropical rain forest regeneration in abandoned pasture: seed rain, seed germination, microclimate, and soil. Biotropica 31(2): 229-242.

Hopkins, M.S. \& Graham, A.W. 1983. The species composition of soil seed banks beneath lowland tropical rainforests in North Queensland, Australia. Biotropica 15: $90-99$.

Hubbell, S.P.; Foster, R.B.; O’Brien, S.T.; Harms, K.E.; Condit, R.; Wechsler, B.; Wright, S.J. \& Loo De Lao, S. 1999. Light-gap disturbances, recruitment limitation, and tree diversity in a neotropical forest. Science 283: 554-557.

Jackson, J.F. 1981. Seed size as a correlate of temporal and spatial patterns of seed fall in a neotropical forest. Biotropica 13(2): 121-130.

Lepsch-Cunha, N.; Kageyama, P.Y. \& Vencovsky, R. 1999. Genetic diversity of Couratari multiflora and Couratari guianensis (Lecythidaceae): consequences of two types of rarity in central Amazonia. Biodiversity and Conservation 8: 1205-1218.

Levey, D.J. 1987. Seed size and fruit-handling techniques of avian frugivores. The American Naturalist 129: 471-485.

Loiselle, B.A.; Ribbens, E. \& Vargas, O. 1996. Spatial and temporal variation of seed rain in a Tropical Lowland Wet Forest. Biotropica 28(1): 82-95.

Marimon, B.S. \& Felfili, J.M. 2000. Distribuição de diâmetros e alturas na floresta monodominante de Brosimum rubescens Taub. na Reserva Indígena Areões, Água BoaMT, Brasil. Revista Árvore 24(2): 143-150.

Marimon, B.S. \& Felfili, J.M. 2001. Ethnobotanical comparison of "Pau Brasil" (Brosimum rubescens Taub.) forests in a Xavante Indian and a Non-Xavante community in eastern Mato Grosso state, Brazil. Economic Botany 55(4):555-569. 
Marimon, B.S.; Felfili, J.M. \& Haridasan, M. 2001a. Studies in monodominant forests in eastern Mato Grosso, Brazil: I. A forest of Brosimum rubescens Taub. Edinburgh Journal of Botany 58(1): 123-137.

Marimon, B.S.; Felfili, J.M. \& Haridasan, M. 2001b. Studies in monodominant forests in eastern Mato Grosso, Brazil: II. A forest in the Areões Xavante Indian Reserve. Edinburgh Journal of Botany 58(3): 483-497.

Martijena, N.E. \& Bullock, S.H. 1994. Monospecific dominance of a tropical deciduous forest in México. Journal of Biogeography 21: 63-74.

Martinez-Ramos, M. \& Soto-Castro, A. 1993. Seed rain and advanced regeneration in a tropical rain forest. Vegetatio 107/108: 299-318.

Morellato, P.C. 1995. As estações do ano na floresta. Pp. 37-41. In: H.F. Leitão Filho \& L.P. Morellato (eds.). Ecologia e preservação de uma floresta tropical urbana - Reserva de Santa Genebra. Campinas, Editora da UNICAMP.

Nascimento, M.T.; Proctor, J. \& Villela, D.M. 1997. Forest structure, floristic composition and soils of an Amazonian monodominant forest on Maracá Island, Roraima, Brazil. Edinburgh Journal of Botany 54(1): 1-38.

Newbery, D.M.; Van Der Burgt, M. \& Moravie, M.-A. 2004. Structure and inferred dynamics of a large grove of Microberlinia bisulcata trees in central African rain forest: the possible role of periods of multiple disturbance events. Journal of Tropical Ecology 20: 131-143.

Runkle, J.R. 1989. Synchrony of regeneration, gaps, and latitudinal differences in tree species diversity. Ecology 70: $546-547$.

SAS Institute. 1999-2000. The SAS System, for Windows. USA, SAS Institute, Carey, NC.

Schluter, D. \& Ricklefs, R.E. 1993. Species diversity in ecological communities. Chicago, University of Chicago Press.
Schupp, E.W. 1992. The Janzen-Connell model for tropical tree diversity: population implications and the importance of spatial scale. The American Naturalist 140(3): 526-530.

Smythe, N. 1970. Relationships between fruiting seasons and seed dispersal methods in a neotropical forest. The American Naturalist 104: 25-35.

Swaine, M.D. \& Whitmore, T.C. 1988. On the definition of ecological species groups in tropical rain forests. Vegetatio 75: 81-86.

Templeton, A.R. \& Levin, D.A. 1979. Evolutionary consequences of seed pools. The American Naturalist 114(2): 232-249.

Tilman, D. 1994. Competition and biodiversity in spatially structured habitats. Ecology 75: 2-16.

Tilman, D. 1999. Diversity by default. Science 283: 495-496.

Torti, S.D.; Coley, P.D. \& Kursar, T.A. 2001. Causes and consequences of monodominance in tropical lowland forests. The American Naturalist 157(2): 141-153.

Walker, L.R. \& Neris, L.E. 1993. Posthurricane seed rain dynamics in Puerto Rico. Biotropica 25(4): 408-418.

Wang, B.C. \& Smith, T.B. 2002. Closing the seed dispersal loop. Trends in Ecology \& Evolution 17(8): 379-385.

Webb, C.O. \& Peart, D.R. 2001. High seed dispersal rates in faunally intact tropical rain forest: theoretical and conservation implications. Ecology Letters 4: 491-499.

White, L.J.T. 1994. Patterns of fruit-fall phenology in the Lopé Reserve, Gabon. Journal of Tropical Ecology 10: 289-312.

Young, K.R.; Ewel, J.J. \& Brown, B.J. 1987. Seed dynamics during forest succession in Costa Rica. Vegetatio 71: 157-173.

Zar, J.H. 1999. Biostatistical Analysis. $4^{\text {th. }}$ ed. New Jersey, Prentice-Hall Inc. 